\title{
La capacitación enfocada en el liderazgo operativo como instrumento para mejorar el servicio de consulta externa en el Hospital del IESS del Cantón Milagro.
}

\section{Training focused on operational leadership as an instrument to improve the external consultation service at the IESS Hospital of Canton Milagro}

Tanya Elizabeth Lopez Macancela. ${ }^{1} \&$ Gonzalo Lenin Serrano Mantilla. ${ }^{2}$

DOI: https://doi.org/10.33262/visionariodigital.v3i2.1..448

\section{Resumen.}

Mediante la presente investigación se busca desarrollar una propuesta de un programa de capacitación enfocada en el liderazgo operativo como instrumento para mejorar el servicio de la consulta externa del Hospital del IESS del Cantón Milagro, todo ello para que se cumpla y favorezca el desempeño de una manera inestimable las actividades y funciones que se encuentran ejecutando cada empleado, para el logro de los objetivos del Centro. En la presente investigación se utilizó tanto del método deductivo como inductivo, mediante los cuales se puedo analizar el estudio del servicio en la consulta externa, y con la aplicación del método comparativo se analizaron los programas de capacitación que contribuyeron a solucionar problemas de efectividad en el desempeño del servicio dado. Como resultado de la investigación se determinó que la evidencia empírica, determina que la falta de liderazgo operativo es el principal obstáculo en la capacitación del personal, otros resultados son que la inadecuada detección de necesidades de capacitación y la carencia de opciones contribuyen a ver la capacitación como un requisito, que, aunque es necesario no resuelve el mejoramiento del servicio de la consulta externa del Hospital del IESS del Cantón Milagro

Palabras clave: Capacitación, Liderazgo, Desempeño, Contribución, Mejoramiento, Efectividad.

\footnotetext{
${ }^{1}$ Instituto Ecuatoriano de Seguridad Social, Guayaquil, Ecuador, tlopezm@iess.gob.ec
}

2 Universidad Estatal de Milagro, Guayaquil, Ecuador gserranom@unemi.edu.ec 


\begin{abstract}
Through the present investigation, we seek to develop a proposal for a training program focused on operational leadership as an instrument to improve the service of the outpatient service of the IESS Hospital of Cantón Milagro, all this to ensure compliance and favor the performance of a Inestimable the activities and functions that are executing each employee, for the achievement of the Center's objectives. In the present investigation, we used both the deductive and inductive method, through which we can analyze the study of the service in the outpatient clinic, and with the application of the comparative method we analyzed the training programs that contributed to solve problems of effectiveness in the performance of the given service. As a result of the investigation it was determined that the empirical evidence determines that the lack of operational leadership is the main obstacle in the training of personnel, other results are that the inadequate detection of training needs and the lack of options contribute to see the training as a requirement, which, although it is necessary, does not resolve the improvement of the service of the outpatient clinic of the IESS Hospital of Cantón Milagro
\end{abstract}

Key words: Training, Leadership, Performance, Contribution, Improvement, Effectiveness

\title{
Introducción.
}

En la presente investigación se busca proveer a los Directivos del HOSPITAL DEL IESS DEL CANTÓN MILAGRO una eficaz herramienta que reúna todas las actividades de capacitación que se requieren para disminuir o eliminar la mayoría de diferencias que pueden tener en su conocimiento los directivos médicos y administrativos, y con ello lograr conocer los requerimientos para priorizar las categorías de urgencia y con ello poder atacarlas mediante el planteamiento de un programa de capacitación que los junte totalmente es por lo que se deberá fomentar una verdadera cultura de capacitación con la cual se asegura el cumplimiento de objetivos, y de las principales prioridades organizacionales y del bienestar en general de la totalidad del personal. Es por ello que para lograr los objetivos planteados al inicio se realiza una investigación que es totalmente sistemática, flexible, analítica y dinámica la cual está enfocada en la calidad y el servicio de la totalidad de funcionarios, siempre tomándose en cuenta la calidad y el servicio de los mismos, para lo que se debe analizar aspectos como es el área a la que pertenecen, los cargos, las funciones entre otros factores.

Para realizar este tipo de investigación se realizaron estudios y teorías en referencia a las áreas que deben estar dentro del área de recursos humanos además de utilizarse métodos de investigación que son tanto participativos y prescriptivos, adicional también se realizaron análisis mediante la técnica de la encuesta que permite conocer las percepciones de las personas a las que va dirigidas. 
$\mathrm{Al}$ estar la presente investigación basada en aspectos teóricos es por lo que existen una serie de conceptualizaciones en referencia a la capacitación que son útiles de ser estudiadas para así poder abordar los temas con los Directivos del hospital para que puedan aceptar la propuesta de capacitaciones desde el punto de vista del liderazgo operativo.

Se considera teóricamente la capacitación o entrenamiento como un proceso para desarrollar las habilidades, actitudes y conocimientos de los funcionarios a los que se les realizará. De acuerdo a lo que se indica por (Diezcanseco, 2015) la capacitación o el entrenamiento constituyen instrumentos que permiten la autorrealización de la educación de las personas que las adquieren, además constituyen el principio en base del cual la comunidad conserva y permite la transmisión de nuevos conocimientos.

De acuerdo a los últimos estudios realizados en referencia a esta modalidad y de acuerdo a Elizondo (2012) en donde se registra un estudio de tipo descriptivo realizado en su país de origen, en donde se pretende alcanzar la planificación mediante un sistema de capacitación permanente, para lo cual se toma una muestra de 100 empleados y 20 jefes departamentales y donde se aplicó el método combinado y se realiza la participación mediante las encuestas de entrevistas lo que genero un resultado del $87 \%$, de empleados y el $95 \%$ de jefes que consideran que la capacitación es una herramienta adecuada para mejorar el desempeño laboral y que mediante lo cual se aumenta la motivación para ser más productivos y eficientes en el trabajo. También en este tipo de estudios los empleados opinaron que lo que es realmente urgente en capacitación está la parte aptitudinal, la cognitiva y la de habilidades, es por ello que se llega a concluir que el plan de capacitación es totalmente beneficioso para cualquier departamento y cualquier institución de salud sin tomar en cuenta ni el sector ni el tipo.

Es por ello que para la presente investigación se ha tomado la iniciativa de realizar un estudio similar al mencionado para conocer las necesidades actuales que tiene el área de consulta externa del hospital del IESS del Cantón Milagro, con el objetivo de mejorar el servicio de consulta externa. Como resultado se ha propuesto el crear un Plan de capacitaciones, mediante diversas estrategias que permitan la correcta implementación, como son los cambios en las estructuras organizativas, por medio de fusiones así como también, el mejoramiento en los recursos humanos a nivel administrativo, para poder mejorar la calidad en los servicios, y la gestión propiamente dicha en la institución médica.

Además, si un empleado que recibe la capacitación necesaria puede realizar mejor su trabajo. Se vuelve más consciente de las prácticas de seguridad y los procedimientos adecuados para las tareas básicas. La capacitación también puede aumentar la confianza del empleado porque tiene una comprensión más sólida de las tareas y actividades del hospital y de las responsabilidades de su trabajo. Esta confianza puede empujarla a rendir aún mejor y pensar en nuevas ideas que la ayuden a sobresalir. 
La capacitación continua también mantiene a sus empleados a la vanguardia de los desarrollos del área de la salud en el hospital. Los empleados que son competentes y que están a la altura de los cambiantes estándares de la atención médica ayudan al hospital a ocupar un puesto como líder y fuerte competidor dentro de las instituciones de salud.

\section{Desarrollo.}

\section{Metodología.}

La metodología de la investigación nos indica el medio más adecuado para realizar un trabajo investigativo, orientado a entender, manejar, y enriquecer los procedimientos teóricos y empíricos de las ciencias, evitando subjetividad, dogmatismo, impresionismo y estereotipos.

Se puede indicar que en toda investigación es muy importante el plantear diversas variables, las cuales permiten relacionar algunos de los conceptos los cuales hacen referencia a las características que el investigador pretende estudiar en el desarrollo investigativo.

En la actualidad existen dos planteamientos paradigmáticos; en el campo de la investigación, que son los estudios cuantitativos y cualitativos. De acuerdo con Bello, Villalobos y Vélez (2005), sostienen que un paradigma, es un conjunto de creencias y actitudes, una visión del mundo que implica explícitamente una metodología determinada; como fuente de métodos, problemas y normas de resolución aceptados por una comunidad científica; que señalan las teorías, hipótesis que deben ser contrastadas, el método y la instrumentación necesarios para la contratación. Cada paradigma o enfoque tiene una concepción diferente de lo que es la investigación; desde: cómo investiga, qué investiga y para qué sirve la investigación. Es necesaria su selección, en virtud de que de acuerdo a la pregunta de investigación y del objetivo general, se diseñaran las estrategias para abordar el objeto o sujeto de estudio.

En la presente investigación se realizará tomando en cuenta el análisis cuantitativo y cualitativo por cuanto se realizará de forma estadística las respuestas de los cuestionarios, así como las opiniones que tengan que informar el personal de las áreas de consulta externa y de talento humano.

Dentro de los métodos a ser utilizados en la presente investigación estarán los siguientes:

El estadístico método con el cual se llegará a establecer la recolección de los datos, así como la incorporación o la afiliación.

Con el método inductivo y deductivo se hallarán la mayoría de las causas y las consecuencias objeto para el estudio y se llegarán a establecer las conclusiones.

Además de estos métodos se tomara en cuenta el analítico que permite realizar la evaluación de los resultados. 
Se analizará además con el método sintético que permite el resumir todos los resultados en referencia a la medición de la variable.

Además, se utilizará el método histórico comparativo que permite sistematizar los conocimientos que son determinantes en referencia al objeto de estudio, así como la comparación de los indicadores que luego determinaran los resultados de las conclusiones.

También hay que tomar en cuenta que se analizarán las muestras del universo establecido en el Cantón Milagro en referencia a los pacientes de esta casa asistencial, tomando una muestra representativa donde se podrá establecer los afiliados activos y donde además se mida la calidad del servicio.

Se realizarán entrevistas a funcionarios, especialistas y usuarios del sistema de seguridad social del cantón y el hospital de Milagro.

\section{Discusión}

Para la presente investigación de este estudio es importante definir los conceptos que se utilizarán mediante los cuales se podrá mantener una armonía en lo referente a la información y además se la podrá estructurar basándose en los autores y temas de propia autoría.

Para ello se podrá tomar en cuenta los conceptos teóricos sobre la capacitación el liderazgo, el liderazgo operativo, así como la mejora continua, también la mejora que se debe dar en el área de trabajo, también se evaluará el significado de la consulta externa puesto que muchas personas no afiliadas no la conocen. Todo este análisis e informaciones constituirán la base para la mejora en el servicio de atención externa por parte del hospital del IESS del Cantón Milagro.

Toda la información obtenida de forma empírica ya sea esta por observación directa o indirecta o con conocimientos teóricos, permiten que se pueda ejecutar planes y programas de capacitación. El equipo de talento humano de una institución de salud es el encargado de estar pendiente y al día en los temas que más le incumben a la institución, en este caso los temas de salud son prioridad y las capacitaciones deben estar al día porque se trabaja con gente y la vida de estas personas muchas veces depende de cómo sean atendidos, para bien o para mal. La inversión también es un tema crítico en este sentido, pero los resultados que puedan generar favorecerán y mejorarán el ambiente y la cultura organizacional.

A continuación, revisaremos y analizaremos algunos conceptos que servirán para el desarrollo de este estudio de investigación.

Además, la experiencia práctica y, más aún, los pacientes que se sienten respetados, están directamente relacionados con la satisfacción de los empleados en el lugar de trabajo. Hacer que los médicos y todos los profesionales de la salud se asocien en este proceso y 
les brinde las herramientas necesarias a través de la educación y la mentoría individual es un paso importante para mejorar la experiencia del paciente.

El monitoreo del desempeño es beneficioso para mejorar la calidad general de la atención al paciente, y se está constantemente implementando nuevas técnicas para proporcionar una experiencia excepcional en los pacientes.

Dentro de esto se debe tomar en cuenta lo siguiente:

1. Fortalecimiento de las instituciones y sistemas de capacitación: ¿cómo podemos fortalecer, estandarizar e institucionalizar los sistemas de capacitación?

Se necesitan vínculos dentro del país entre los sistemas de aprendizaje para fortalecer e institucionalizar los sistemas de capacitación. Los programas de capacitación en servicio deben ser proporcionados por instituciones locales para aumentar la apropiación y la sostenibilidad de la capacitación. La estandarización de los materiales y el programa de capacitación pueden maximizar los recursos existentes para la capacitación para capacitación y mejorar la efectividad y la eficiencia de la capacitación.

2. Coordinación de la capacitación: ¿qué mecanismos se pueden utilizar para coordinar mejor la capacitación? ¿Qué características son las más deseables en un sistema de información / seguimiento de entrenamiento?

Con frecuencia, los programas de capacitación en servicio capacitan a las mismas personas una y otra vez, mientras que otros no están capacitados en absoluto. Un sistema para el seguimiento de los cursos de capacitación y los participantes no solo mejoraría la eficiencia, la eficacia y la sostenibilidad de la capacitación, sino que también ayudaría a garantizar un acceso equitativo a la capacitación de todos los trabajadores de la salud.

3. Continuo aprendizaje a partir del servicio previo al servicio: ¿Qué roles pueden desempeñar las diferentes partes interesadas para garantizar el aprendizaje continuo entre la educación previa al servicio y la capacitación en el servicio?

Las instituciones de servicios previos pueden estar en el centro de una estructura de capacitación en servicio coordinada ofreciendo cursos directamente o acreditando a grupos como asociaciones profesionales para brindar capacitación. Este modelo puede ayudar a garantizar la coherencia entre el contenido de la capacitación en servicio y en servicio y permitir que las capacitaciones en servicio cuenten para los programas de grado ofrecidos por las instituciones en servicio.

4. Diseño y entrega de capacitación: una revisión reciente de la literatura sugiere que la capacitación basada en el lugar de trabajo más corta, repetida y con gran simulación puede ser más efectiva. Dado que el sistema actual está orientado hacia la capacitación en grupo y en el aula, ¿cómo podemos implementar metodologías más efectivas? 
Los eventos de capacitación deben estar vinculados con el desarrollo profesional continuo de la salud, y se deben explorar oportunidades adicionales para la capacitación en el lugar de trabajo utilizando metodologías alternativas que respalden un proceso continuo de control de calidad.

5. Apoyo para el aprendizaje: ¿Cómo preparamos, apoyamos e incentivamos a los ocupados trabajadores de la salud para que proporcionen orientación o apoyo basados en el lugar de trabajo a otros?

Un entorno en el trabajo que permite al empleado utilizar nuevos conocimientos y habilidades es esencial para traducir el aprendizaje en una mejor calidad de los servicios y resultados de salud.

6. Evaluación y mejora de la capacitación: ¿Cómo puede fortalecerse la evaluación de los resultados de la capacitación en servicio para informar la mejora en la capacitación? ¿Cuáles son algunos de sus éxitos y algunas de sus necesidades para identificar los resultados de sus programas de capacitación?

La determinación metodológica de la multitud de factores que influyen en los resultados de los programas de capacitación requiere mucho tiempo, reflexión y recursos. El marco y las herramientas de evaluación de la capacitación pueden ayudar a abordar este desafío.

\section{Conceptos básicos de capacitación}

Muchos de estos conceptos que se presentarán a continuación, aportarán una idea más clara de las definiciones de diferentes autores. Desde la lectura de estos conceptos, se logrará formar una definición más completa de lo que se busca en este estudio y servirán como referente para el desarrollo del Programa de capacitación para el Hospital General de Milagro. Entre los conceptos más exactos a lo que se busca, se han identificado los siguientes:

"La capacitación consiste en proporcionar a los empleados nuevos o actuales, las habilidades necesarias para desempeñar su trabajo. Proceso de enseñanza de las aptitudes básicas que los nuevos empleados necesitan para realizar su trabajo”. (Carrillo, 2014)

"Actitudes del personal en conductas produciendo un cambio positivo en el desempeño de sus tareas. El objeto es perfeccionar al trabajador en su puesto de trabajo". (Benavides, 2010)

"La capacitación se considera como un proceso a corto plazo, en que se utiliza un procedimiento planeado, sistemático y organizado, que comprende un conjunto de acciones educativas y administrativas orientadas al cambio y mejoramiento de conocimientos, habilidades y actitudes del personal, a fin de propiciar mejores niveles de desempeño compatibles con las exigencias del puesto que desempeña, y por lo tanto 
posibilita su desarrollo personal, así como la eficacia, eficiencia y efectividad empresarial a la cual sirve". (Moran, 2012)

Para varios autores, las definiciones de capacitación o entrenamiento podrían tener significados distintos, muchos especialistas tenían en cuenta que el entrenamiento debía adecuase para cada persona según cargo, experiencia profesional, tiempo en la institución médica, desempeño y un sin número de cualidades adicionales, pero esto no mide la fuerza laboral y mucho menos quien debe tomar realmente un entrenamiento. Desde siempre se ha entendido por entrenamiento, un conjunto de actividades para desarrollar algún tipo de destrezas que van enfocadas a un puesto exacto de trabajo y el cual requerirá algún conocimiento adicional. Sin embargo, actualmente esto es considerado una forma para el desarrollo de habilidades blandas y duras para las personas. En estos términos, se puede decir que es una forma eficaz para agregarle valor a las personas que trabajan dentro de una compañía y que esto enriquece su trabajo y logra de manera más rápida los objetivos de la institución.

De acuerdo con las definiciones anteriores, se puede llegar a la conclusión de que la capacitación es la herramienta que tienen muchísimas organizaciones para mejorar y empoderar a sus colaboradores, a sus clientes internos, mejorando sus competencias personales y profesionales, contribuyendo en gran medida a la compañía y favoreciendo la rentabilidad para todas las partes interesadas.

Fases de cambio mediante el entrenamiento o capacitación

Todas las instituciones de salud y organizaciones médicas esperan que, al contratar un colaborador, este tenga todas las actitudes y capacidades para ingresar directamente a hacer ganar a la institución. Este concepto o pensamiento no está bien planteado, ya que esto depende mucho de la experiencia y de las capacidades que tenga este colaborador para el giro del negocio que tiene la institución médica. En muchos casos, esto falla y en consecuencia se tiene que invertir millones en los empleados, pero al final del día, toda esa información y dinero invertido, será parte de la contribución para lograr los objetivos del hospital.

Según varios autores, existen 4 fases de cambio para el comportamiento mediante el entrenamiento:

1. Transmisión de información:

Es uno de los elementos más importantes para lograr con éxito un entrenamiento o capacitación para un grupo o un solo empleado. Dentro de las actividades que deben tomarse en cuentan están: la correcta distribución de la información, la programación del tiempo, los temas y las horas de receso para poder asimilarlo todo. En muchas ocasiones la información puede ser la que necesita el colaborador directamente y en otras ocasiones, indirectamente se le enseña sus nuevas responsabilidades.

2. Desarrollo de habilidades: 
En este punto se recalca todas las destrezas y actitudes relacionadas con la forma en la que se desenvuelve un colaborador dentro de la institución de salud. Debe tomarse en cuenta que siempre deben estar las capacitaciones orientadas a lo que se necesita en el hospital, ya sea en actividades, aptitudes, formas de desempeñar un trabajo específico. El objetivo de esto es capacitar y ejecutar actividades en pro de la institución

3. Desarrollo o modificación de actitudes:

Este punto se refiere al manejo de conflictos y el cambio de la actitud de las personas que colaboran dentro de una institución de salud. Todo con el fin de facilitar las relaciones interpersonales y lograr relaciones a largo plazo. Esto también, fortalece la confianza, la motivación, mejora el sentido común y la percepción que se tiene al momento de tomar una decisión sin interferir en los intereses del hospital.

4. Desarrollo de conceptos:

Las capacitaciones que se entregan a los colaboradores de un grupo específico también van en función de los conceptos o nuevas capacidades teóricas que requieran los empleados para diversos temas dentro de la compañía. En de suma importancia enfocarse en la conceptualización y mostrar la mejor forma de que se aprender. Se debe incluir a mandos altos, medios y bajos. Ya que un concepto nuevo debe socializarse en general a toda institución de salud.

Las fases que se mencionan podrían utilizarse por separado o en conjunto para explicar varios temas o comunicar no sólo conceptos sino formas intrínsecas de hacer algo para beneficio del hospital.

Gráfico N1. Plan estratégico

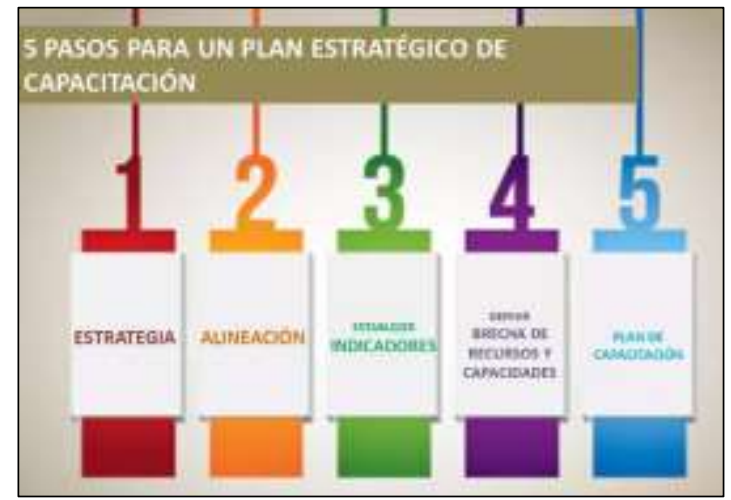

Fuente: Elaboración propia.

\section{Objetivos del entrenamiento o capacitación}

Después de revisar estas fases en los comportamientos que podrían adoptar las personas que reciben un entrenamiento específico, se debe conocer los objetivos que deben cumplir restos pilares, entre ellos tenemos los siguientes de acuerdo a varios autores: 
1. "Preparar al personal para la ejecución inmediata de las diversas tareas del cargo." (Alban)

2. "Proporcionar oportunidades para el desarrollo personal continuo, no solo en su cargo actual, sino también en otras funciones en las cuales puede ser considerada la persona.

3. Cambiar la actitud de las personas, bien sea para crear un clima más satisfactorio entre los empleados, aumentar su motivación o hacerlos más receptivos a las técnicas de supervisión y gerencia". (Espinoza)

Con las capacitaciones, se puede fortalecer los conocimientos y actitudes de los miembros de la institución para mejorar el desempeño de su vida laboral y personal. Finalmente, gracias a esto se pueden mejorar los objetivos y las responsabilidades de cada colaborador.

Gráfico N2. Proceso de capacitación

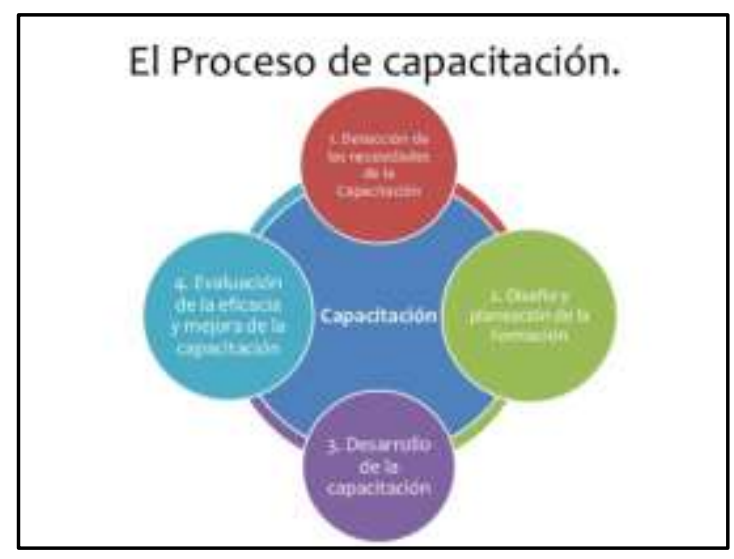

Fuente: Elaboración propia del autor.

Proceso del entrenamiento o capacitación

Se puede plantear un proceso en el cual el entrenamiento llegue al colaborador de forma eficaz y mucho más eficiente, en la que sólo se necesite explicar estos temas una vez teniendo toda la atención de ellos.

Chiavenato, menciona ya un proceso programado que tiene ventajas y beneficia a la organización, entre estos pasos se encuentras:

1. Diagnostico situacional.

2. Lista de necesidades para los colaboradores del área determinada o de toda la institución.

3. Programación del día, de las horas, de los recursos para atender de forma completa todas las necesidades.

4. Definir el alcance del entrenamiento.

5. Especificar tiempos para la implementación y para la ejecución de las actividades que resulten.

6. Evaluación de resultados.

7. Aplicación de la mejora continua. 
El entrenamiento o capacitación es responsabilidad de un departamento específico y puede asumir la responsabilidad para retroalimentar a toda la organización siempre y cuando conozca sus principales insuficiencias. Es ideal crear un modelo descentralizado para que equilibre a la organización y encuentre realmente quienes son los más necesitados de estas implementaciones.

Inventario de necesidades de entrenamiento o capacitación

Conocido también como el inventario de necesidades o de diagnóstico para que los entrenamientos se cumplan con éxito. Se debe tener también clara la misión y la visión, los objetivos y todo aquello en lo que su finalidad sea el cumplimiento de los objetivos de la institución.

Otro autor menciona lo siguiente, basado en el tema de estudio actual:

"la primera etapa del entrenamiento es la evaluación de necesidades de entrenamiento de la organización, que no siempre son muy claras y se deben diagnosticar a partir de ciertos censos e investigaciones internas capaces de localizarlas y descubrirlas. Las necesidades de entrenamiento son las carencias de preparación profesional de las personas, es decir, la diferencia entre lo que una persona debería saber y hacer y aquello que realmente sabe y hace" (Carrillo, 2014)

Cuando se identifican las carencias, la eliminación es mucho más rápida y benéfica para los empleados, ya que, al tener un mejor ambiente, se tiene también, un clima laboral excepcional para trabajar. Estos inventarios pueden realizarse en varios niveles, a continuación, se mencionan:

1. Análisis total de la organización.

2. Análisis del Recurso Humano

3. Análisis de las Operaciones y de las tareas de la institución.

1. Análisis total de la institución:

"implica el estudio de la institución como un todo, incluyendo al ambiente socioeconómico y tecnológico en el cual está situada la organización. Este análisis ayuda a responder la incógnita acerca de lo que debe instruir en términos de un plan, y establece la filosofía del entrenamiento para toda la institución. Consiste en determinar en donde deberá hacer énfasis el entrenamiento, en este sentido el análisis organizacional deberá verificar todos los factores (planes, fuerza laboral, eficiencia organizacional, clima organizacional, etc.), evaluar los costos implicados y los beneficios esperados del entrenamiento, en comparación con otras estrategias capaces de alcanzar los objetivos institucionales, y determinar así la política global relacionada con el entrenamiento. El entrenamiento se desarrolla de acuerdo con las necesidades de la institución. A medida que la organización crece, sus necesidades cambian y, por consiguiente, el entrenamiento deberá responder a la nueva demanda. Las necesidades deben inventariarse, determinarse 
e investigarse con cierta periodicidad para establecer, a partir de ellas, los programas adecuados para satisfacerlas de manera conveniente". (Ambrossio, 2009)

El entrenamiento debe ser una actividad continua y constante para que el desempeño de las personas sea el adecuado. Se debe recordar que la base principal de estos programas son las personas y su actitud para asimilar todo lo que se les quiere ofrecer de conocimientos.

\section{Análisis del recurso humano}

Para analizar el recurso humano se procura verificar la suficiencia de los colaboradores y que tan dispuestos están a aceptar información nueva ya sea cuantitativa o cualitativamente. Para la fuerza laboral es de suma importancia que la institución funcione correctamente, para esto se deben tomar en cuenta algunos puntos importantes para los entrenamientos que se lleven a cabo:

1. Cantidad de empleados de acuerdo con el departamento.

2. Edad de cada empleado de acuerdo con el departamento.

3. Puntaje del nivel de compromiso que posee con la institución.

4. Puntaje en el nivel de conocimiento exigido para cada empleado.

5. Actitudes de cada colaborador de acuerdo con el departamento en relación al trabajo y al sentido de pertenencia con la institución.

6. Desempeño cuantitativo y cualitativo de cada colaborador.

7. Nivel de habilidades para cada empleado

8. Potenciales para definir si se puede reclutar internamente.

9. Potenciales para definir si se puede reclutar externamente.

10. Definir el tiempo de entrenamiento necesario para el equipo laboral.

11. Definir el tiempo de entrenamiento necesario para nuevas personas reclutadas.

12. Determinar los índices de ausentismo.

13. Determinar los índices de la rotación del personal.

14. Describir y analizar los cargos.

Cada uno de estos se deben estar analizando continuamente, de esto depende que la organización cumpla sus metas y en función de los plazos establecidos lo logre de manera eficaz y eficiente.

3. Análisis de las Operaciones y tareas de la empresa

Para este enfoque, es limitada la información de la lista de necesidades, ya que se debería efectuar el análisis del cargo dependiendo de la persona ocupante y de los requisitos el cargo exige a la persona que estará al frente. La organización y los colaboradores deben tomar en consideración las actividades y los cargos actuales para los que las personas fueron entrenadas. Este análisis sirve para comprobar las habilidades y conocimientos y sobre todo la personalidad de los ocupantes de estos puestos. Este estudio debe tomar en consideración el área del hospital en la cual se implementarán todas estas capacitaciones y entrenamientos. Este punto consta de algunos datos, a continuación, se nombran:

- Patrón adecuado de desempeño para los cargos. 
- Definición de tareas y actividades que componen el cargo.

- El cómo hacer para desempeñar de forma correcta cada tarea y mantener los patrones exigidos para el desempeño.

- Finalmente, se mencionan las habilidades, actitudes y conocimientos para desempeñar las tareas.

Para el análisis de las operaciones, se comprende de un proceso de descomposición de los cargos, esto para verificar las habilidades, las cualidades personales y para definir las responsabilidades de estos agentes en cada ámbito del desarrollo de sus funciones.

Identificación de necesidades de entrenamiento

De acuerdo con varios autores, este inventario se puede definir de la siguiente manera:

"El inventario de necesidades de entrenamiento es un diagnóstico que debe basarse en información pertinente, gran parte de la cual debe ser agrupada de modo sistemático, en tanto que otra reposa disponible en manos de ciertos administradores de línea." (Cardenas)

"El inventario de necesidades de entrenamiento es una responsabilidad de línea y una función de staff: corresponde al administrador de línea la responsabilidad de detectar los problemas provocados por la carencia de entrenamiento." (Carrillo, 2014)

Los medios principalmente usados para realizar el inventario de las necesidades de entrenamiento son algunos de los que se nombran a continuación:

- Evaluación del desempeño:

Estas evaluaciones sirven para demostrar que tanto el empleado conoce y ha puesto en práctica de lo explicado y desarrollado en las capacitaciones. Esto es muy importante para saber en qué se debe mejorar en futuros entrenamientos.

- Observación:

La directa y la indirecta, de eso depende como el sentido común soporte la idea de las capacitaciones y que tanto se refuercen para mejora individual y colectiva de los empleados de una institución pública o privada.

- Cuestionarios:

Desarrollar medios para evaluar el conocimiento de los colaboradores y que quede en el tiempo para futuros entrenamientos.

- Solicitud de responsables:

Los líderes de las áreas son los responsables de medir el rendimiento de sus empleados y determinar si es necesaria una capacitación de algún tema en particular para ellos.

- Entrevistas con los responsables: 
Se debe evaluar si lo que solicitan los responsables de área es verdaderamente lo que necesita la institución para fortalecer su alcance de objetivos.

- Reuniones con los departamentos entrenados:

Estas reuniones fortalecen los lazos entre departamentos y mejoran las relaciones interpersonales entre ellos.

- Exámenes a los colaboradores:

Estos exámenes son necesarios para conocer las necesidades básicas y complementarias de los colaboradores y poder ejercer normativas para este aspecto.

- Análisis de cargos:

Es vital para las organizaciones realizar por lo menos un análisis de cargos cada año, ya que esto sirve para fortalecer el cargo de la persona y a la persona que se designe para el mismo.

- Informes periódicos:

Los informes periódicos reflejan una idea de cómo se encuentra la institución en este ámbito, es por eso que es de suma importancia que todo esté de acuerdo a las medidas y objetivos que se toman.

Programación del entrenamiento o capacitación

Una vez que ya se tiene una idea de la lista de necesidades existentes, hay que preguntarse cuál es la prioridad para la organización, a continuación, se muestran algunas preguntas que se pueden realizar para definir este punto:

- ¿Cuáles son las necesidades más importantes de la institución?

- ¿En qué estamos fallando a simple vista?

- ¿Del reporte de quejas, que área es la más afectada?

- ¿Cuáles son las causas?

- ¿Es personal nuevo o con antigüedad?

- ¿Es necesario aplicar indicadores?

- ¿En cuánto tiempo se puede solucionar?

- ¿Cuánto tiempo tiene establecido la compañía para solucionar esto?

- ¿Es una necesidad permanente o temporal?

- ¿Puede ser costoso?

- ¿Existen opciones para impartir este entrenamiento?

Estas preguntas ayudarán a la persona encargada a resolver de manera ágil el inconveniente de definir las prioridades. Por otro lado, los costos y los tiempos a pesar de que se pueden definir se deben dejar para discutirlos con los jefes de área y los colaboradores que manejen estos rubros directamente.

A través de la historia se ha observado la forma como ha evolucionado el concepto y la importancia del factor humano dentro de las organizaciones, como una estrategia clave para el éxito y el crecimiento de esta. Gracias a ello, las instituciones han desarrollado planes de mejoramiento con el fin de conservar, descubrir y aprovechar habilidades que 
tal vez el mismo hombre no sabía de sí mismo, creando un equilibrio de crecimiento y beneficio mutuo, entre los empleados y el hospital.

\section{Conclusiones.}

- Como resultado de las investigaciones realizadas, se toma en cuenta que las faltas del liderazgo operativo constituyen el obstáculo a nivel general dentro del Hospital del IESS del Cantón Milagro para el mejoramiento en el servicio de consulta externa, además de medios para dar capacitaciones a nivel general, se pude indicar que:

a) No existen muchas opciones de capacitaciones en este sector.

b) Se puede especificar que la capacitación no es la acorde para el puesto que se encuentra desempeñando el empleado en la institución.

c) Se puede indicar a la capacitación como uno de los requisitos para que se promuevan a otros cargos.

d) Se deberá respetar el programa de capacitación luego de que este se encuentre elaborado.

e) Es necesario establecer limitantes para los jefes de área en referencia a la solicitud de los cursos de capacitación del personal.

f) Es requerimiento limitar el desarrollo laboral del hospital del IESS.

g) Se precisa ser más rápido y eficaz el conseguir los objetivos del hospital del IESS.

h) No se encuentra al momento que exista un cumplimiento en lo referente al Sistema Integrado de Gestión de Calidad en el Hospital del IESS del Cantón Milagro.

- En definitiva, se determina que es muy difícil lograr la implementación de este tipo de iniciativas por cuanto existe la falta de continuidad en los funcionarios que son los responsables de aplicar este tipo de programas por cuanto los cambios de administración en el hospital del IESS conllevan cambios de funcionarios.

- Además, la capacitación presenta una excelente oportunidad para ampliar la base de conocimientos de todos los empleados del hospital, pero muchos empleadores consideran que las oportunidades de desarrollo son caras. Los empleados del hospital también pierden tiempo de trabajo mientras asisten a las sesiones de capacitación, lo que puede demorar la finalización de los proyectos. A pesar de los posibles inconvenientes, la capacitación y el desarrollo brindan a la institución de salud en su conjunto ya los empleados individuales beneficios que hacen que el costo y el

\section{Referencias Bibliográficas.}

Abdala, E. (2001). Experiencias de capacitación laboral de jóvenes en América Latina, Ultima década. (online) 2001, vol. 9 n. 14 pp.113-135. Recuperado el 29 de octubre de 2010, de http://www.scielo.cl/scielo.php? script=sci_arttext\&pid=s0718-223620010000 100007 \&lng=es\& nrm=iso

ADEXNEWS. (30 de 9 de 2014). Adexperu boletin de noticias $N^{o} 680$. Obtenido de http://www.adexperu.org.pe/BoletinesD/Prensa/BPrensa.asp?bol=1945\&cod=10 7 
Alban, N. (s.f.). Oportunidades de mejora para los entrenamientos. 2013.

Ambrossio, J. (2009). Definiciones de capacitaciones para organigramas. La paz.

Asamblea Nacional. (13 de julio de 2011). Constitución de la República 2008. Obtenido de Asamblea Nacional: www.oas.org/juridico/PDFs/mesicic4_ecu_const.pdf

Asamblea Nacional. (13 de junio de 2011).

www.oas.org/juridico/PDFs/mesicic4_ecu_const.pdf. Obtenido de Constitución 2008: www.oas.org/juridico/PDFs/mesicic4_ecu_const.pdf

Briceño, A. (1985). Derecho individual del Trabajo. México. Harla. 95 \#106 / Año 39 Centro de investigación e innovación tecnológica. (2009). Manual de Sistema Integrado de Gestión de la Calidad. México. Instituto Politécnico Nacional.

Benavides, L. (2010). Actitudes personales para el ser humano.

Chiavenato, I. (2006). Introducción a la teoría general de la administración. México. Mc Graw Hill.

Chiavenato, I. (2007). Administración de recursos humanos. México. Mc Graw Hill.

Cardenas, N. (s.f.). La evaluación del desempeño. 2009.

Carrillo, M. B. (2014). Programa para el Líderazgo.

CONSTITUCION DE LA REPUBLICA DEL ECUADOR 2008. (s.f.).

De luna, A. (2008). Capital humano. México.

Diezcanseco, M. (2015). El aprendizaje por medio de los planes de capacitación. 37.

Donabedian, A. (s.f.). Universidad de Michigan. Obtenido de https://www.esan.edu.pe/apuntes-empresariales/2016/05/los-diferentesconceptos-de-calidad-en-salud/

El Comercio. (29 de septiembre de 2015). adipiscor.com. Obtenido de adipiscor.com: https://www.adipiscor.com/como/807/Afiliacion-al-IESS-de-las-Trabajadorasno-Remun

El Comercio. (26 de enero de 2016). https://www.adipiscor.com/como/807/Afiliacional-IESS-de-las-Trabajadoras-no-Remun. Obtenido de adipiscor.com: https://www.adipiscor.com/como/807/Afiliacion-al-IESS-de-las-Trabajadorasno-Remun

Elizondo, C. (2012). Capacitaciones permanentes para mejorar la productividad.

Espinoza, M. J. (s.f.). Procesos de entrenamiento o capacitación en una organización. 2010. 
Ibañez, J. L. (2011). Capacidades y habilidades de los sistemas de capacitación. Colombia.

INEC. (2014). www.Inec .gov.ec.Ecuador en cifras. Obtenido de http://www.ecuadorencifras.gob.ec/

Instituto Ecuatoriano de Seguridad Social. (2005). Obtenido de https://www.iess.gob.ec/es/inst-quienes-somos

Moran, B. S. (2012). La fuerza laboral en la organización.

Nacional, A. (5 de octubre de 2015). http://www.asambleanacional.gob.ec/es/contenido/afiliacion-de-lastrabajadoras-no-remuneradas-del-hogar. Obtenido de asambleanacional.gob.ec: http://www.asambleanacional.gob.ec/es/contenido/afiliacion-de-lastrabajadoras-no-remuneradas-del-hogar

Reyes, R. (2003). Comunicación y mercadotecnia política. Mexico: Limusa.

Trillas. Dessler, G. (2001). Administración de Personal. México. Pearson Educación.

Trillas. Granier, C. (2008). Organizaciones que aprenden. Recuperado el 20 de marzo de 2008, de http://top5km.blogspot.com/2009_10_01_archive.html

Santiago. Mideplan. Gómez, R. (2006). Administración de los recursos humanos en instituciones educativas. México.

valverde, J. V. (11 de Mayo de 2016). Los diferentes conceptos de Calidad en la Salud. Obtenido de https://www.esan.edu.pe/apuntes-empresariales/2016/05/losdiferentes-conceptos-de-calidad-en-salud/ 


\section{Para citar el artículo indexado.}

Lopez T. \& Serrano G., (2019). La capacitación enfocada en el liderazgo operativo como instrumento para mejorar el servicio de consulta externa en el Hospital del IESS del Cantón Milagro. Revista electrónica Visionario Digital 3(2), 266-283. Recuperado desde: http://visionariodigital.org

\section{Ligital}

El artículo que se publica es de exclusiva responsabilidad de los autores y no necesariamente reflejan el pensamiento de la Revista Ciencia Digital.

El articulo queda en propiedad de la revista y, por tanto, su publicación parcial y/o total en otro medio tiene que ser autorizado por el director de la Revista Ciencia Digital.
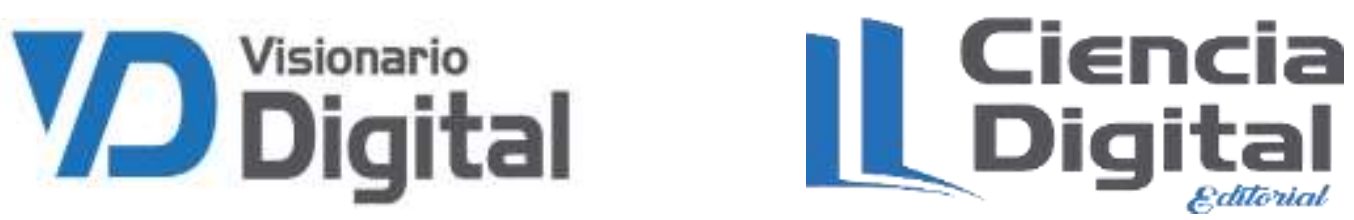